\title{
A NOTE ON THE DIRECTION OF ENERGY MOVEMENT IN WAVENUMBER OF A TWO-LAYER MODEL
}

\author{
HAL G. MARSHALL * \\ Space Physics Research Laboratory, Department of Atmospheric and Oceanic Science, \\ The University of Michigan, Michigan, MI (U.S.A.) \\ (Received April 10, 1985; revised October 17, 1985; accepted June 13, 1986)
}

\section{ABSTRACT}

Marshall, H.G., 1986. A note on the direction of energy movement in wavenumber of a two-layer model. Dyn. Atmos. Oceans, 10: 253-257.

An equation on the constrained direction of energy movement in wavenumber is derived for the two-layer, f-plane, quasi-geostrophic system.

\section{INTRODUCTION}

The majority of energy is constrained to move toward smaller wavenumber in an inviscid dispersionless two-dimensional fluid (Onsager, 1949; Fjortoft, 1953; Merilees and Warn, 1975). Charney (1971) proved that theorems for a multi-layer geostrophic fluid are of the same form as those of a two-dimensional fluid. Charney also showed upscale movement of energy in a three-dimensional wavenumber.

Rhines (1977) described and interpreted energy movements in the wavenumber domain from adiabatic inviscid numerical two-layer simulations. In his pioneering work, however, he did not use the perspective of the vertical modes and this unnecessarily complicates some simple points. Salmon (1980) used the vertical modes for both a qualitative discussion via triad arguments, intuition and closure modeling with the two-layer model. Hoyer and Sadourny (1982) extensively examined the two-layer model with closure methods. This latter study requires a detailed knowledge of constrained turbulence closures.

\footnotetext{
* Current address: Laboratory for Atmospheric Research, Washington State University, Pullman, WA 99164-2730, U.S.A.
} 
Merilees and Warn (1975) suggested a simple method (due to G. Batchelor) to discern the direction of the cascades of energy in an inviscid two-dimensional fluid. The relevant conserved quantities of the barotropic vorticity equation are

$$
\frac{\partial}{\partial t} \int_{0}^{\infty} E_{k} \mathrm{~d} k=0
$$

and

$$
\frac{\partial}{\partial t} \int_{0}^{\infty} k^{2} E_{k} \mathrm{~d} k=0
$$

where $\psi$ is the streamfunction, $J$ the Jacobian, $E_{k}$ the energy density at wavenumber $k$, and $k^{2} E_{k}$ the enstrophy at wavenumber $k$. An infinite domain is chosen. Assume that

$$
\frac{\partial}{\partial t} \int_{0}^{\infty}\left(k-k_{\mathrm{c}}\right)^{2} E_{k} \mathrm{~d} k>0
$$

where $k_{\mathrm{c}}$ is the centroid of the spectrum $\left(k_{\mathrm{c}}=\int_{0}^{\infty} k E_{k} \mathrm{~d} k / \int_{0}^{\infty} E_{k} \mathrm{~d} k\right)$. From eq. 3 the constraints (1) and (2) demand

$$
\frac{\partial}{\partial t} k_{c}<0
$$

Thus energy must move upscale, that is, to a smaller $k$ for the conservation principles to hold. (4) is a necessary, but not sufficient, condition for satisfying (1) and (2). Spreading of an initially narrow wavenumber peak is intuitively reasonable and is repeatedly found in numerical simulations of both two-dimensional and quasigeostrophic non-narrow wavenumber peaks (e.g., Marshall et al., 1983). The spreading can be thought of as a result of two opposing visual attributes of two-dimensional and quasigeostrophic turbulence: (1) the thinning and teasing out of contours of vorticity or potential vorticity by smaller scales (e.g., Rhines, 1977); (2) the inhibition of this teasing out by large scale fields. Which effect is largest is not important in deciding the defocusing assumption. This is simply decided by the opposite tendencies of these two effects.

For the two-layer model, in modal form, this method gives a simple way of obtaining a constraint on the direction of the energy cascade. The advantage of this method is that no knowledge of the triads (Marshall and Chen, 1982), or the complex details of a constrained turbulence closure is required.

\section{SCALE CHANGES OF ENERGY IN A SIMPLE BAROCLINIC MODEL}

The simplest nonlinear system to include baroclinicity is the quasigeostrophic two-layer model on the f-plane. The potential vorticity equations 
for this system are (e.g., Salmon, 1980)

$$
\begin{aligned}
& \frac{\partial \nabla^{2}}{\partial t} \psi+J\left(\psi, \nabla^{2} \psi\right)+J\left(\tau, \nabla^{2} \tau\right)=0 \\
& \frac{\partial}{\partial t} \nabla^{2} \tau-R^{2} \frac{\partial \tau}{\partial t}+J\left(\psi, \nabla^{2} \tau-R^{2} \tau\right)+J\left(\tau, \nabla^{2} \psi\right)=0
\end{aligned}
$$

where $\psi$ and $\tau$ are the barotropic and baroclinic modal streamfunctions, respectively. $R=\sqrt{2} / L$ where $L$ is the Rossby deformation radius and $J$ is the Jacobian.

Using Parseval's identity, one can separate the constraint of conservation for total energy into three logical but not distinct parts and the enstrophy in two parts (e.g., Salmon, 1980) for each wavenumber

$K_{\psi k}=|\overrightarrow{\mathbf{k}}|^{2} \Psi_{k}^{*} \Psi_{k}$

$K_{\tau k}=|\overrightarrow{\mathbf{k}}|^{2} \tau_{k}^{*} \tau_{k}$

$A_{k}=R^{2} \tau_{k}^{*} \tau_{k}$

which are the barotropic kinetic, baroclinic kinetic and available potential energy densities. The baroclinic energy is the sum of these two latter energies $(6 b)$ and $(6 c) .()^{*}$ is the complex conjugate. Likewise potential enstrophy, $\varepsilon_{\psi k}=|\overrightarrow{\mathbf{k}}|^{2} K_{\psi k}$

$\varepsilon_{\tau k}=\left(|\overrightarrow{\mathbf{k}}|^{2}+R^{2}\right)\left(K_{\tau k}+A_{k}\right)$

Note the baroclinic potential enstrophy (7) is not just the wavenumber squared multiplied by the total energy.

Define the baroclinic, barotropic and total energy centroids as follows:

$k_{\mathrm{c} \tau}=\int_{0}^{\infty} k\left(K_{\tau k}+A_{k}\right) \mathrm{d} k / E_{R}$

$k_{\mathrm{c} \psi}=\int_{0}^{\infty} k K_{\psi k} \mathrm{~d} k / E_{T}$

and

$k_{\mathrm{c}}=k_{\mathrm{c} \tau}+k_{\mathrm{c} \psi}$

the defocusing assumption can be stated as

$$
\frac{\partial}{\partial t} \int_{0}^{\infty}\left(k-k_{\mathrm{c}}\right)^{2}\left(K_{\psi k}+K_{\tau k}+A_{k}\right) \mathrm{d} k>0
$$

The defocusing assumption can be motivated (see introduction). Simulation under a variety of conditions and parameters suggests this assumption is generally true (e.g., Marshall et al., 1983). For simplicity define $\alpha$ as the 
ratio of baroclinic to total energy and $C(\psi \rightarrow \tau)$ as the rate of conversion of barotropic to baroclinic energy. With the conservation relations as well as (10)

$$
-\frac{\partial k_{\mathrm{c}}^{2}}{\partial t}>\frac{\partial R^{2}}{\partial t} \alpha+\frac{R^{2}}{E_{\mathrm{T}}} C(\psi \rightarrow \tau)
$$

First consider the situation where the Rossby deformation wavenumber $\left(R^{2}\right)$ is independent of time. The total centroid of energy must decrease in horizontal wavenumber when the conversion of energy from barotropic to baroclinic form is positive. In Charney's (1971) theory the cascade seeks the lowest three-dimensional wavenumber and is forbidden to go higher in the three-dimensional wavenumber index. In order for $k_{\mathrm{c}}$ to decrease, energy must at least go to the next lower vertical mode. The two-layer model then converts baroclinic energy to the lower vertical mode-the barotropic mode. Also note the effect of the Rossby deformation wavenumber in modulating how much the conversion of barotropic to baroclinic energy is a constraint on the horizontal wavenumber movement and vice versa. This modulation is independent of the conversion's internal dependence on the Rossby deformation radius (e.g., Marshall et al., 1983).

In the baroclinic unstable growth of a spectrum of waves the horizontal nonlinear interactions would be less constrained by positive conversion of baroclinic to barotropic energy in accord with eq. 12. After the baroclinic instability is released this conversion would become small and the interactions more constrained to upscale cascading.

There is no need here to assume artificial boundary conditions at the bottom or top of the models such as isothermal or isentropic surfaces. The temperature at any point in the two-layer model is interpreted as the vertically averaged temperature and no a priori assumption is required as a boundary condition for temperature. Thus Charney's results are much more general than indicated by his restrictive isothermal boundary conditions.

Consider the situation when conversion term $C(\psi \rightarrow \tau)$ is zero. Note the decrease in the total centroid must be greater than the increase of the Rossby deformation wavenumber modulated by the fraction of energy in baroclinic form. The rate of change of $R^{2}$ would have to be small such that eqs. $5 \mathrm{a}$ and $5 \mathrm{~b}$ are not violated.

Also, since $k_{\mathrm{c}}$ is made up of a linear combination of the barotropic and baroclinic centroids and $k_{\mathrm{c}}$ appears in (11) as a square, a troublesome cross-term clouds the inequality when examining these centroids separately. A slightly different formulation of the spreading assumption

$$
\frac{\partial}{\partial t} \int_{0}^{\infty}\left\{\left(k-k_{\mathrm{c} \tau}\right)^{2}\left(K_{k \tau}+A_{R}\right)+\left(k-k_{\mathrm{c} \psi}\right)^{2} K_{\psi}\right\} \mathrm{d} k>0
$$


results in

$-\frac{\partial k_{\mathrm{c} \psi}^{2}}{\partial t}-\frac{\partial k_{\mathrm{c} \tau}^{2}}{\partial t}>\frac{\partial R^{2}}{\partial t}+\frac{R^{2}}{E_{t}} C(\psi \rightarrow \tau)$

which gives no cross term.

\section{CONCLUDING REMARKS}

The direction of cascading for energy in the two-layer quasigeostrophic f-plane is examined. A simple 'five-line proof' produces some conditions for this conservative system. These relations apply to the centroids of the energies; thus, they do not say that no part of the energy can violate these inequalities but that the mean energy cannot. The constraint of upscale cascading is found to be in accord with that of the study of Charney (1971) without having to assume the same horizontal boundary conditions. The simplicity of the relations (11) and (13) is a direct consequence of the relation between the two-layer baroclinic vertical mode and the thermal wind.

\section{ACKNOWLEDGEMENTS}

The author was partly supported by NASA Grant NAGW-176 and NSF Grant OCE8305648. The comments of J. Boyd were helpful as was a point by $\mathrm{P}$. Rhines.

\section{REFERENCES}

Charney, J.G., 1971. Geostrophic turbulence. J. Atmos. Sci., 28: 1087-1095.

Fjortoft, R., 1953. On the changes in the spectral distribution of kinetic energy for two-dimensional, nondivergent flow. Tellus, 5: 225-230.

Hoyer, J. and Sadourny, H., 1982. Closure modeling of fully developed baroclinic instability. J. Atmos. Sci., 39: 707-721.

Marshall, H.G. and Chen, T.-C., 1982. Baroclinic nonlinear exchanges of energy and potential enstrophy in the quasi-geostrophic two-layer model. Geophys. Astrophys. Fluid Dynamics, 22: 71-84.

Marshall, H.G., Chen, T.-C. and Tribbia, J.J., 1983. A numerical study of the nonlinear cascading of atmospheric baroclinic and barotropic flow with a two-layer quasi-geostrophic model. NSF Technical Report. Also, Ph.D. Thesis. Available from University Microfilms, Ann Arbour, MI.

Merilees, P.E. and Warn, H., 1975. On energy and entrophy exchanges in two-dimensional non-divergent flow. J. Fluid Mech., 69: 625-630.

Onsager, L., 1949. Statistical hydrodynamics. Nuevo Cumento supp., 6: 287-297.

Rhines, P.B., 1977. The dynamics of unsteady currents. In: The Sea. Wiley and Sons, New York, pp. 189-318.

Salmon, R., 1980. Baroclinic instability and geostrophic turbulence. Geophys. Astrophys. Fluid Dynamics, 15: 167-211. 